\title{
Muon Spin Rotation and Relaxation in the Superconducting Ferromagnet UCoGe
}

\author{
A. de Visser, ${ }^{1, *}$ N. T. Huy, ${ }^{1, \dagger}$ A. Gasparini, ${ }^{1}$ D. E. de Nijs,${ }^{1}$ D. Andreica, ${ }^{2, \ddagger}$ C. Baines,${ }^{2}$ and A. Amato ${ }^{2}$ \\ ${ }^{1}$ Van der Waals-Zeeman Institute, University of Amsterdam, Valckenierstraat 65, 1018 XE Amsterdam, The Netherlands \\ ${ }^{2}$ Laboratory for Muon-Spin Spectroscopy, Paul Scherrer Institute, CH-5232 Villigen, Switzerland \\ (Received 11 September 2008; published 22 April 2009)
}

\begin{abstract}
We report zero-field muon-spin rotation and relaxation measurements on the superconducting ferromagnet UCoGe. Weak itinerant ferromagnetic order is detected by a spontaneous muon-spin precession frequency below the Curie temperature $T_{C}=3 \mathrm{~K}$. The $\mu^{+}$precession frequency persists below the bulk superconducting transition temperature $T_{\mathrm{sc}}=0.5 \mathrm{~K}$, where it measures a local magnetic field $B_{\text {loc }}=$ $0.015 \mathrm{~T}$. The amplitude of the $\mu \mathrm{SR}$ signal provides unambiguous proof for ferromagnetism present in the whole sample volume. We conclude ferromagnetism coexists with superconductivity on the microscopic scale.
\end{abstract}

DOI: 10.1103/PhysRevLett.102.167003

PACS numbers: 74.70.Tx, 75.30.Kz, 76.75.+i

Recently, it was shown [1] that UCoGe belongs to a new family of ferromagnetic metals which become superconducting at low temperatures. One of the most intriguing properties of these superconducting ferromagnets (SCFMs) is that both ordering phenomena coexist. Such a coexistence is incompatible with the standard BCS theory for superconductivity (SC), because the ferromagnetic (FM) exchange field prevents phonon-mediated formation of singlet Cooper pairs [2]. However, for weak itinerant ferromagnets, like UCoGe, an alternative route is offered by spin fluctuation models [3,4], in which critical magnetic fluctuations near a magnetic phase transition can mediate unconventional SC by pairing electrons in a spin triplet state. Superconductivity not mediated by phonons [5] attracts wide interest as it plays a central role in the quest to unravel high temperature SC in the cuprates [6] and the oxypnictides [7] discovered recently. The SCFMs discovered so far are $\mathrm{UGe}_{2}$ (under pressure) [8], UIr (under pressure) [9], URhGe [10], and UCoGe. The coexistence of itinerant FM and SC in these compounds marks a clear distinction with other families of superconductors, such as Chevrel phases [11] and borocarbides [12], in which localmoment (anti)ferromagnetism and SC exist, but expel each other. In the case of $\mathrm{UGe}_{2}$ [13] and URhGe [14] experimental evidence is at hand that SC is driven by critical magnetic fluctuations near a magnetic transition between two polarized phases. In contrast, UCoGe may present the first example of SC stimulated by critical fluctuations associated with a FM quantum critical point.

FM order in UCoGe with a Curie temperature $T_{C}=3 \mathrm{~K}$ was first observed by magnetization $M(T, B)$ measurements on polycrystalline samples [1]. The small (polycrystalline averaged) ordered moment $m_{0}=0.03 \mu_{B}$ and the low coercive field of $\sim 1 \mathrm{mT}$ indicate magnetism is weak. The analysis of the magnetization by means of Arrott plots shows UCoGe is an itinerant FM. This is further corroborated by specific heat data, which show the entropy associated with the magnetic transition is small $(0.3 \%$ of $R \ln 2)$.
Subsequent $M(T, B)$ data taken on single crystals reveal a strong uniaxial magnetic anisotropy: the moment $m_{0}=$ $0.07 \mu_{B}$ points along the orthorhombic $c$ axis [15]. In the FM state SC occurs with a resistive transition temperature $T_{\mathrm{sc}}=0.8 \mathrm{~K}$ for the best polycrystalline samples. Measurements of the upper critical field $B_{c 2}$ provide evidence for unconventional SC, characterized by an equalspin pairing triplet state and a SC gap function with point nodes along the direction of the uniaxial moment [15].

Although FM order is a robust property of our poly- and single-crystalline samples, it has not been probed directly via magnetization below $1.8 \mathrm{~K}$ [1]. Moreover, the $M(T, B)$ data alone cannot exclude that the small moment is due to a reduced volume part of the sample exhibiting FM order with a larger magnetic moment. Evidence obtained so far for the coexistence of SC and FM stems mainly from macroscopic measurements, notably thermal expansion [1]. The sizes of the steps in the coefficient of linear thermal expansion at $T_{C}$ and $T_{\mathrm{sc}}$ indicate magnetism and $\mathrm{SC}$ occur in the whole sample, however, again, reduced volume fractions cannot be ruled out. Therefore, it is of paramount importance to investigate the magnetic response of UCoGe on a microscopic scale. The $\mu$ SR technique is extremely suitable for this purpose [16]. Positive muons $\mu^{+}$, when implanted in the sample, act as a sensitive probe of the local field, which permits one to discern magnetically inequivalent sample regions. In this Letter we present a $\mu$ SR study on UCoGe which provides unambiguous proof for magnetic order in the whole sample volume. FM coexists with SC below $T_{\mathrm{sc}}$. Our work is the first $\mu \mathrm{SR}$ study conducted on a SC itinerant FM below its SC transition temperature. The muon response in the SC state provides evidence for a spontaneous vortex phase in zero field. Furthermore, our results provide insight into the intricate problem of how the superconductor accommodates the FM structure.

A batch of polycrystalline UCoGe was prepared as described in Ref. [1]. Measurements of the electrical re- 
sistivity, $R(T)$, show the residual resistance ratio $\mathrm{RRR}=$ $R(300 \mathrm{~K}) / R(1 \mathrm{~K}) \approx 30$ and the $\mathrm{FM}$ and SC transitions occur at 3.0 and $0.8 \mathrm{~K}$ (onset), respectively [see Fig. 3(a)]. Zero-field (ZF) $\mu$ SR experiments were performed at the Paul Scherrer Institute in the general purpose spectrometer (GPS) using a ${ }^{4} \mathrm{He}$ flow cryostat in the temperature range $T=1.6-10 \mathrm{~K}$ and in the low temperature facility (LTF) using a dilution refrigerator for $T=0.02-5.5 \mathrm{~K}$. Two identical $\mu$ SR samples were prepared. Each sample consisted of four thin slices (thickness $0.8 \mathrm{~mm}$, area $6 \times$ $10 \mathrm{~mm}^{2}$ ) that were cut from the annealed button by spark erosion. The surface layer, defected by spark erosion, was removed by polishing. The total sample area was $12 \times$ $20 \mathrm{~mm}^{2}$ and matched the cross-sectional area of the muon beam in order to eliminate contributions from the sample holder. One sample was measured in the GPS and the other in the LTF. In addition we carried out ZF $\mu$ SR experiments on polycrystalline $\mathrm{URh}_{0.4} \mathrm{Co}_{0.6} \mathrm{Ge}$, which has a Curie temperature $T_{C}=20.0 \mathrm{~K}$ [17] and a (polycrystalline averaged) ordered moment $m_{0}=0.22 \mu_{B}$ [18].

ZF $\mu$ SR is a well-known technique to study magnetically ordered phases. Polarized muons are implanted into a sample where their spins $\mathbf{S}_{\mu}\left(S_{\mu}=1 / 2\right)$ precess in the local magnetic field $\mathbf{B}_{\text {loc }}$ until they decay. $\mathbf{S}_{\mu}(t)$ is monitored through the decay positron. By measuring the asymmetric distribution of emitted positrons the time evolution of the polarization $\mathbf{P}_{\mu}(t)$ can be deduced. $P_{\mu}(t)$ is defined as the projection of $\mathbf{P}_{\mu}(t)$ along the direction of initial polarization $\quad P_{\mu}(t)=\mathbf{P}_{\mu}(t) \cdot \mathbf{P}_{\mu}(0) / P_{\mu}(0)=G(t) P_{\mu}(0)$, where $G(t)$ reflects the normalized muon-spin autocorrelation function $G(t)=\left\langle\mathbf{S}_{\mu}(t) \cdot \mathbf{S}_{\mu}(0)\right\rangle / S_{\mu}(0)^{2}$. The muon depolarization function $G(t)$ extracted from the $\mu$ SR spectra contains the information on the averaged value, distribution, and time evolution of the internal magnetic fields and is to be compared with selected theory functions.

A typical ZF $\mu$ SR spectrum on UCoGe in the paramagnetic state at $T=5.5 \mathrm{~K}$ is shown in Fig. 1(a). This spectrum is best described by the standard Kubo-Toyabe function:

$$
G_{\mathrm{KT}}(t)=\frac{1}{3}+\frac{2}{3}\left(1-\Delta_{\mathrm{KT}}^{2} t^{2}\right) \exp \left(-\frac{1}{2} \Delta_{\mathrm{KT}}^{2} t^{2}\right) .
$$

The Kubo-Toyabe function describes the muon depolarization due to an isotropic Gaussian distribution of static internal fields centered at zero field. $\Delta_{\mathrm{KT}}=\gamma_{\mu} \sqrt{\left\langle B^{2}\right\rangle}$ is the Kubo-Toyabe relaxation rate, with $\gamma_{\mu}$ the muon gyromagnetic ratio $\left(\gamma_{\mu} / 2 \pi=135.5 \mathrm{MHz} / \mathrm{T}\right)$ and $\left\langle B^{2}\right\rangle$ the second moment of the field distribution. Spectra taken in the paramagnetic phase show $\Delta_{\mathrm{KT}}$ is $0.30 \pm 0.01 \mu \mathrm{s}^{-1}$ and is independent of temperature. This indicates the depolarization is not due to electronic magnetic moments, but rather due to static nuclear moments of the ${ }^{59} \mathrm{Co}$ atoms $\left(\mu_{\mathrm{Co}}=5.23 \mu_{N}\right.$, the spin $I=7 / 2$ and the abundance is $100 \%)$.

In the FM state, a clear spontaneous $\mu^{+}$precession frequency $\nu$ is observed. A typical spectrum, taken at $T=$
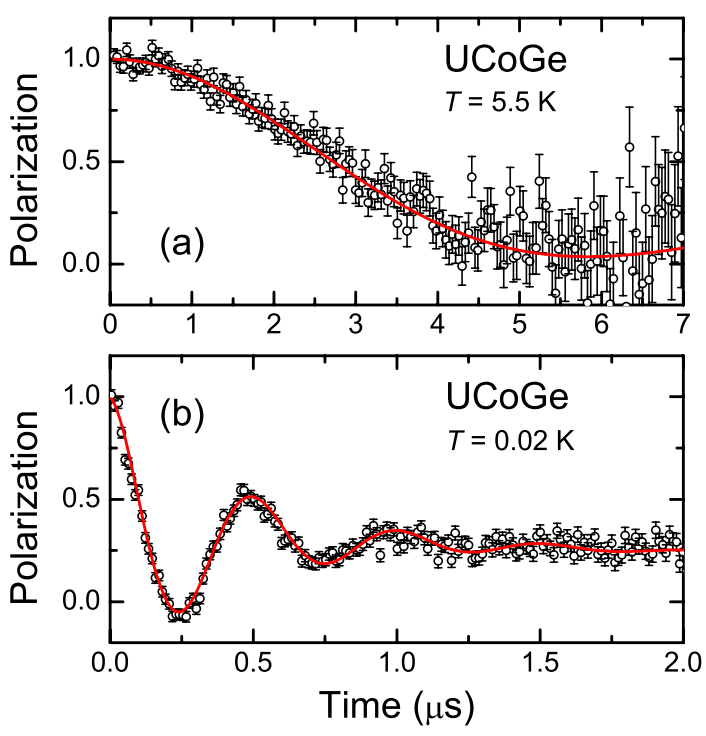

FIG. 1 (color online). Typical zero-field $\mu$ SR spectra in polycrystalline UCoGe: (a) in the paramagnetic phase at $T=5.5 \mathrm{~K}$ [solid line fit to Eq. (1)], and (b) in the ferromagnetic phase at $T=0.02 \mathrm{~K}$, well below the superconducting transition temperature [solid line fit to Eq. (2)].

$0.02 \mathrm{~K}$, is shown in Fig. 1(b). This response has an electronic origin and is best described by the depolarization function of an isotropic polycrystalline magnet with a Lorentzian field distribution:

$$
G_{M}(t)=\frac{1}{3} \exp \left(-\lambda_{1} t\right)+\frac{2}{3} \exp \left(-\lambda_{2} t\right) \cos (2 \pi \nu t+\phi) .
$$

The first term corresponds to an average of $1 / 3$ of the muons that sense a local field parallel to their initial polarization and therefore do not precess, where $\lambda_{1}$ is a measure for the internal spin dynamics perpendicular to the muon spin. The " $2 / 3$ term" probes the local field which results in a precession of the muon spin with frequency $\nu$ ( $\phi$ is a phase factor) and $\lambda_{2}$ is a measure for both static and dynamical spin effects. By fitting the spectrum taken at $T=0.02 \mathrm{~K}$ [Fig. 1(b)] to Eq. (2) we obtain the spontaneous frequency $\nu=1.972 \pm 0.004 \mathrm{MHz}$, and the damping rates $\lambda_{1}=0.152 \pm 0.006 \mu \mathrm{s}^{-1}$ and $\lambda_{2}=$ $2.372 \pm 0.004 \mu \mathrm{s}^{-1}$. The measured value of $\lambda_{1}$ confirms the " $1 / 3$ term" is due to the sample and muons do not stop in the sample holder. As will be demonstrated below, the spontaneous $\mu^{+}$precession frequency characterizes the weak itinerant FM order. The observed frequency corresponds to a local field $B_{\text {loc }} \sim 0.015 \mathrm{~T}$ at the muon localization site.

In order to investigate the evolution of the magnetic volume fraction in the temperature regime of the FM transition we have fitted the $\mu$ SR spectra to a twocomponent depolarization function:

$$
G(t)=A_{\mathrm{magn}} G_{M}(t)+A_{\mathrm{para}} G_{\mathrm{KT}}(t) .
$$

Here $A_{\text {magn }}$ and $A_{\text {para }}$ represent the volume fractions of the ferro- and paramagnetic phases, respectively, with 
normalization $A_{\text {magn }}+A_{\text {para }}=1$. In Fig. 2 we show the deduced temperature variation $A_{\text {magn }}(T)$ and $A_{\text {para }}(T)$. The magnetic transition is relatively broad in our polycrystalline samples. Below $T \sim 1.5 \mathrm{~K} A_{\text {magn }}(T) \rightarrow 1$ and magnetism is present in the whole sample volume. The $\mathrm{ZF}$ asymmetry is within error bar equal to the asymmetry measured in transverse field $(\mathrm{TF}=50 \mathrm{G})$ at temperatures of $5.5 \mathrm{~K}$ (LTF) and $10 \mathrm{~K}$ (GPS), which confirms the full signal amplitude is involved.

In Fig. 3 we show $\nu(T)$, which tracks the magnetization $M(T)$ [1]. The $\nu(T)$ data can be well fitted to the phenomenological order-parameter function $\nu(T)=$ $\nu_{0}\left(1-\left[T / T^{*}\right]^{\alpha}\right)^{\beta}$. The spontaneous frequency vanishes near $T^{*}=3.02 \mathrm{~K} \approx T_{C}$ and $\nu_{0}=1.98 \mathrm{MHz}$. Notice the critical exponent $\alpha=2.3$ deviates from the standard value $3 / 2$ for spin waves, while $\beta=0.4$ is larger than the theoretical value 5/16 for a 3D Ising-like FM [19].

In order to further explore the $\mu$ SR spectra in the magnetic state we have carried out ZF muon-spin rotation experiments on UCoGe doped with Rh. Upon substituting $\mathrm{Rh}$ for Co the Curie temperature and the ordered moment steadily increase and reach the values $T_{C}=20.0 \mathrm{~K}$ [17] and $m_{0}=0.22 \mu_{B}$ [18] for 40 at. \% Rh. ZF $\mu$ SR spectra on polycrystalline $\mathrm{URh}_{0.4} \mathrm{Co}_{0.6} \mathrm{Ge}$ were collected in the GPS for $T=5-25 \mathrm{~K}$. In the FM phase the muon depolarization is described by Eq. (2), but best fits were obtained with a Gaussian damping factor $\exp \left(-\frac{1}{2} \sigma_{G}^{2} t^{2}\right)$ for the $2 / 3$ term. From the amplitude of the signal we conclude magnetism is observed in the whole sample volume. The main feature of the spectra is the spontaneous $\mu^{+}$precession frequency below $T_{C}$, which attains a value of $14.1 \mathrm{MHz}$ at the lowest temperature (see inset of Fig. 3). $\nu(T)$ follows an orderparameter variation with $T^{*}=20.1 \mathrm{~K} \approx T_{C}$. The values $\alpha=2.2$ and $\beta=0.4$ are similar to the ones derived from the magnetization [18], and identical, within the error bars, to the values obtained for UCoGe. The precession frequency $\nu(0)$ is a factor $\sim 7$ larger than the one of UCoGe

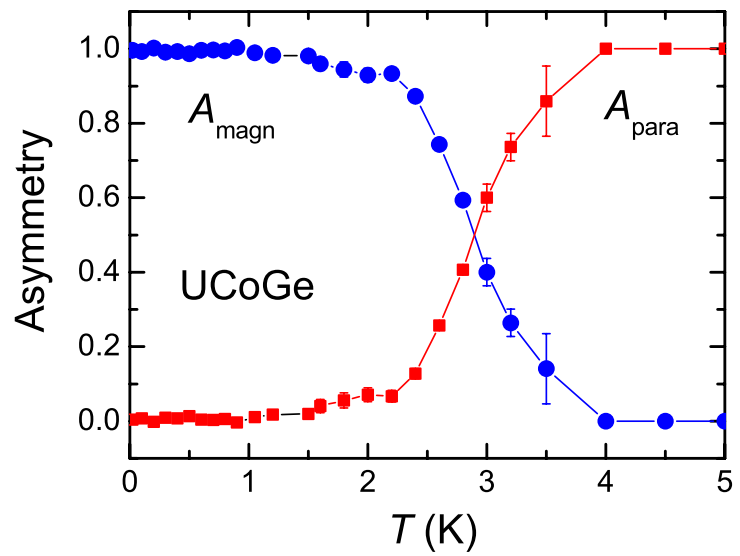

FIG. 2 (color online). Temperature variation of the normalized asymmetries of the ferromagnetic and paramagnetic phase in UCoGe. Below $\sim 1.5 \mathrm{~K}$ magnetism is observed in the whole sample volume. The solid lines are to guide the eye. and scales with the ratio of the ordered moments $(\sim 7)$ deduced from the dc magnetization [18]. We conclude the analysis of the ZF $\mu$ SR spectra of $\mathrm{URh}_{0.4} \mathrm{Co}_{0.6} \mathrm{Ge}$ corroborates the results for UCoGe.

Next we discuss the possible muon localization site. UCoGe crystallizes in the orthorhombic TiNiSi type structure $[20,21]$ (space group $P_{n m a}$ ), where the $\mathrm{U}, \mathrm{Co}$, and Ge atoms occupy the $4 \mathrm{c}$ sites (Wyckoff notation). The most probable localization sites are the high symmetry interstitial $4 \mathrm{a}$ and $4 \mathrm{~b}$ positions. By calculating $\Delta_{\mathrm{KT}}$ due to the ${ }^{59} \mathrm{Co}$ nuclear moments $\left(T>T_{C}\right)$ one can in principle determine the stopping site [22]. From a search along high symmetry directions in the crystal leads we tentatively assign the $\mu^{+}$ localization site to the $4 \mathrm{a}$ position, where $\Delta_{\mathrm{KT}}=$ $0.34 \mu \mathrm{s}^{-1}$ [23], close to the experimental value of $0.30 \pm$ $0.01 \mu \mathrm{s}^{-1}$. The local field in the FM phase (in ZF) is given by $B_{\text {loc }}=B_{\text {dip }}+B_{c}+B_{L}$, where $B_{\text {dip }}$ is the dipolar sum of the ordered moments, $B_{c}$ is the contact hyperfine field, and $B_{L}=\mu_{0} M_{s} / 3$ the Lorentz field with $M_{s}$ the saturation magnetization (see, for example, Ref. [24]). For a precise determination of the different terms Knight shift experiments on a single crystal are required. However, dipolar calculations yield the following estimates: with $m_{0}=0.07 \mu_{B} \| c$ [15], which we assume is located at the $\mathrm{U}$ atoms, we derive for the $4 \mathrm{a}$ site $B_{\text {dip }}=0.015 \mathrm{~T}$, $B_{L}=0.005 \mathrm{~T}$, and by comparing to the experimental

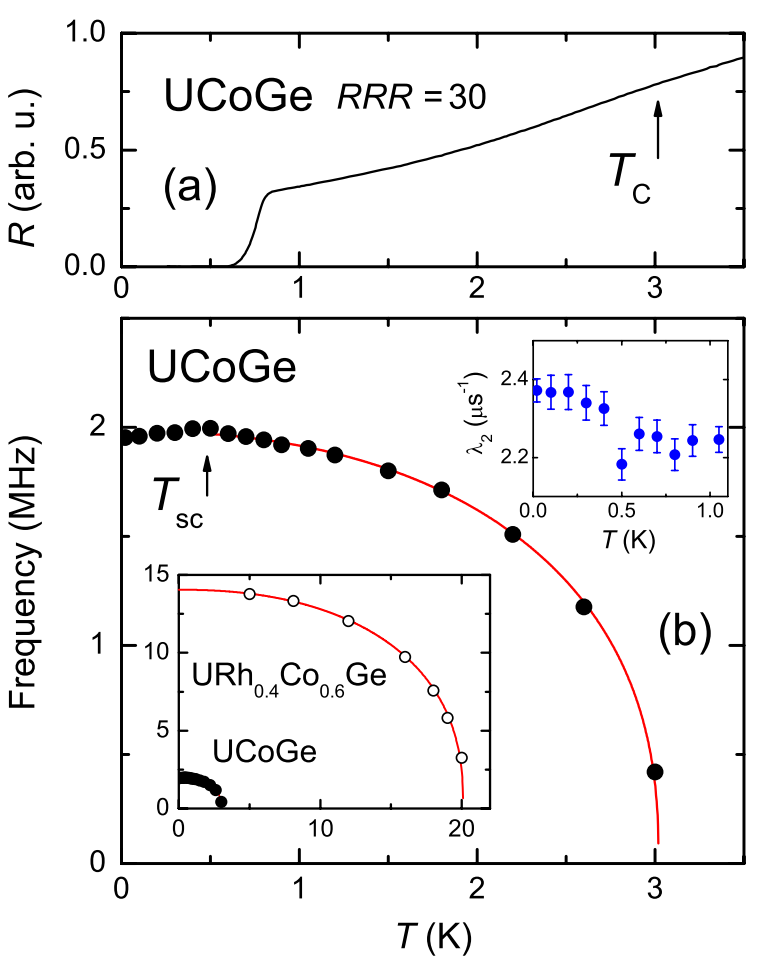

FIG. 3 (color online). (a) Resistivity versus temperature of polycrystalline UCoGe. (b) Spontaneous muon precession frequency $\nu(T)$ of UCoGe and (lower inset) $\mathrm{URh}_{0.4} \mathrm{Co}_{0.6} \mathrm{Ge}$. The solid lines represent fits to a phenomenological order-parameter function (see text). Notice the $2 \%$ decrease of $\nu(T)$ below the bulk $T_{\text {sc }}$ of $0.5 \mathrm{~K}$ [1]. Upper inset: $\lambda_{2}(T)$ of UCoGe. 
value $B_{\text {loc }}$ of $0.015 \mathrm{~T}$, a negative contact field $B_{c}=$ $-0.005 \mathrm{~T}$.

We now have a detailed understanding of the ZF muonspin rotation data and proceed to make a number of important conclusions. First of all, the data provide unambiguous proof for ferromagnetism present in the whole sample volume. The spontaneous $\mu^{+}$precession signal persists with the same asymmetry below the SC transition temperature (see Fig. 3). Thus FM and SC coexist. Interestingly, in the SC state the precession frequency shows a small decrease of about $2 \%$. This decrease is measured by the whole muon ensemble. It provides a first indication of the interplay of magnetism and SC in UCoGe. The decrease of $\nu$ below $T_{\mathrm{sc}}$ is possibly due to induced screening currents that lead to a small reduction of the local field. On the other hand, the damping factor $\lambda_{2}$ slightly increases when passing through $T_{\text {sc }}$ (see Fig. 3). Such a broadening of the field distribution is expected when a spontaneous vortex phase [25] is formed. This indicates the local field is larger than the lower critical field $B_{c 1}$ and the sample is always in the mixed state. Our results also shed light on the intricate question of how the SC state accommodates FM order. The strong uniaxial magnetic anisotropy [15] excludes the formation of a modulated or spiral magnetic structure when passing through $T_{\mathrm{sc}}$, as observed in $\mathrm{ErRh}_{4} \mathrm{~B}_{4}$ [26]. This is corroborated by the minor changes in the $\mu \mathrm{SR}$ spectra below $T_{\mathrm{sc}}$. It has been proposed that the SC transition should be accompanied by a redistribution of FM domains [27,28], however, such a redistribution cannot be detected in our polycrystal data. $\mu$ SR experiments on single crystals are needed to elucidate these issues further.

We emphasize the FM state is observed in zero applied magnetic field. Recent magnetization measurements on single crystals $(R R R=20)$ led to the claim that a magnetic field of the order of a few $\mathrm{mT}$ is needed to stabilize FM order [29]. This is at variance with our results [15] and indicates metallurgy of $\mathrm{UCoGe}$ is an important issue. This might also be of relevance for understanding recent Co NMR and NQR data obtained on powdered samples [30]. The analysis reveals a two-component nuclear spin relaxation rate $1 / T_{1}$, yielding evidence for an inhomogeneous sample and a SC volume fraction of $30 \%$.

In summary, we have used the $\mu$ SR technique to investigate the weak itinerant FM phase in UCoGe. Below $T_{C}=$ $3 \mathrm{~K}$ a spontaneous $\mu^{+}$precession frequency appears, which probes a local field $B_{\text {loc }}$ of $0.015 \mathrm{~T}$ when $T \rightarrow 0$. Muons most likely localize at the 4a site, as deduced from calculations of the Kubo-Toyabe relaxation rate. The $\mu^{+}$ precession frequency persists below $T_{\mathrm{sc}}$, where FM and SC coexist on the microscopic scale.

This work was part of the research program of the Dutch Foundation FOM. Part of this work was carried out at the Swiss Muon Source $\mathrm{S} \mu \mathrm{S}$ (PSI, Villigen, Switzerland) with support of the European Commission under the 6th
Framework Programme through the COST Action P16 ECOM and the Key Action Strengthening the European Research Area, Research Infrastructures (Contract No. RII3-CT-2003-505925).
*a.devisser@uva.nl
${ }^{\dagger}$ Current address: Hanoi Advanced School of Science and Technology, Hanoi University of Technology, 1 Dai Co Viet, Hanoi, Vietnam.
"Current address: Faculty of Physics, Babes-Bolyai University, 400084 Cluj-Napoca, Romania.

[1] N. T. Huy et al., Phys. Rev. Lett. 99, 067006 (2007).

[2] N. F. Berk and J. R. Schrieffer, Phys. Rev. Lett. 17, 433 (1966).

[3] D. Fay and J. Appel, Phys. Rev. B 22, 3173 (1980).

[4] G. G. Lonzarich, in Electron: A Centenary Volume, edited by M. Springford (Cambridge Univ. Press, Cambridge, 1997), Chap. 6.

[5] P. Monthoux, D. Pines, and G. G. Lonzarich, Nature (London) 450, 1177 (2007).

[6] J. G. Bednorz and K. A. Müller, Z. Phys. B 64, 189 (1986).

[7] Y. Kamihara et al., J. Am. Chem. Soc. 130, 3296 (2008).

[8] S. S. Saxena et al., Nature (London) 406, 587 (2000).

[9] T. Akazawa et al., J. Phys. Condens. Matter 16, L29 (2004).

[10] D. Aoki et al., Nature (London) 413, 613 (2001).

[11] Ø. Fischer, Appl. Phys. 16, 1 (1978).

[12] R. J. Cava et al., Nature (London) 367, 252 (1994).

[13] C. Pfleiderer and A.D. Huxley, Phys. Rev. Lett. 89, 147005 (2002).

[14] F. Lévy et al., Science 309, 1343 (2005).

[15] N. T. Huy et al., Phys. Rev. Lett. 100, 077002 (2008).

[16] A. Amato, Rev. Mod. Phys. 69, 1119 (1997).

[17] S. Sakarya et al., J. Alloys Compd. 457, 51 (2008).

[18] N. T. Huy, Ph.D. thesis, University of Amsterdam, 2008; N. T. Huy and A. de Visser, Solid State Commun. 149, 703 (2009).

[19] J.E. Essam and M.E. Fisher, J. Chem. Phys. 38, 802 (1963).

[20] B. Lloret, Ph.D. thesis, University Bordeaux I, 1988.

[21] F. Canepa et al., J. Alloys Compd. 234, 225 (1996).

[22] A. Schenck, Muon Spin Rotation Spectroscopy (Adam Hilger, Bristol, 1985).

[23] For the $4 \mathrm{~b}$ site $\Delta_{\mathrm{KT}}=0.20 \mu \mathrm{s}^{-1}$. The second moment calculation includes quadrupolar coupling effects. We assume a radial electric field gradient and neglect the small contribution from nuclear moments at Ge atoms.

[24] A. Schenck and F. N. Gygax, Handbook of Magnetic Materials, edited by K.H.J. Buschow (North-Holland, Amsterdam, 1995), Vol. 9, Chap. 2.

[25] M. Tachiki et al., Solid State Commun. 34, 19 (1980).

[26] S. K. Sinha et al., Phys. Rev. Lett. 48, 950 (1982).

[27] P. W. Anderson and H. Suhl, Phys. Rev. 116, 898 (1959).

[28] E. B. Sonin, Phys. Rev. B 66, 100504(R) (2002).

[29] J. Poltierová Vejprová, J. Pospísuil, and V. Sechovský, arXiv:0806.3561v1.

[30] T. Ohta et al., J. Phys. Soc. Jpn. 77, 023707 (2008). 